\title{
Light Scattering from Polymer Films Having Optically Anisotropic Rodlike Texture. II. Principle to Estimate Its Size*
}

\author{
Takeji Hashimoto, Kiyonaga Yamaguchi, \\ and Hiromichi KAWAI \\ Department of Polymer Chemistry, Faculty of Engineering, \\ Kyoto University, Kyoto 606, Japan.
}

(Received March 25, 1977)

\begin{abstract}
The theory for estimating the size of isotropic rodlike particles dispersed randomly in space was extended to anisotropic rodlike particles. The scattering from a random assembly of the anisotropic rods is more complex than that of the isotropic rods in that the angular dependence of the scattering depends upon the manner of orientation of the optical axes of anisotropic scattering elements as well as size of the rod. In order to evaluate size of the rod, one has to separate these two contributions. It is shown that relative intensity distribution of the intensity difference at $\mu=0^{\circ}$ and $45^{\circ}$, i.e., $\left(I_{\mathrm{H}_{\mathrm{v}}}\right)_{\mathrm{dif}}=\left|I_{\mathrm{H}_{\mathrm{v}}}\left(\mu=0^{\circ}\right)-I_{\mathrm{H}_{\mathrm{v}}}\left(\mu=45^{\circ}\right)\right|$ depends only upon size, and is identical to that of isotropic rods of the same size at large scattering angles. The intensity $\left(\boldsymbol{I}_{\mathrm{H}_{\mathrm{V}}}\right)_{\text {dif }}$ may be then used to evaluate size of the anisotropic rodlike particle.

KEY WORDS Light Scattering / Polymer Films / Anisotropic Rod-

like Particles / Guinier Plot / Size Evaluation /
\end{abstract}

The technique of light scattering from polymeric solid has been developed to characterize the nature of crystalline superstructure of a size comparable to the wavelength of visible light. The scattering from spherulites which are most commonly observed in polyolefin films crystallized from isotropic melts has been well analyzed by Stein and coworkers. ${ }^{1-3}$ The analyses yielded information on average size of the spherulites, degree of spherulite perfection (including both internal disorders and truncation of the shape), volume fraction of spherulites, crystallinity within and outside the spherulites.

The average size of the spherulites can be evaluated easily and rapidly by observing $\theta_{\max }$, the scattering angle at which the $H_{\mathrm{V}}$ spherulitic scattering intensities become maximum (see Figure 1) and by applying the equation given by Stein-Rhodes, ${ }^{1}$

* Presented in part at 25th Polymer Symposium, Society of Polymer Science, Japan, 1976, Nagoya University, Japan.

Reference 5 is designated as Part $I$ of this series hereafter.

$$
\frac{4 \pi}{\lambda} R \sin \frac{\theta_{\max }}{2}=4.1
$$

where $R$ is the radius of average size of the

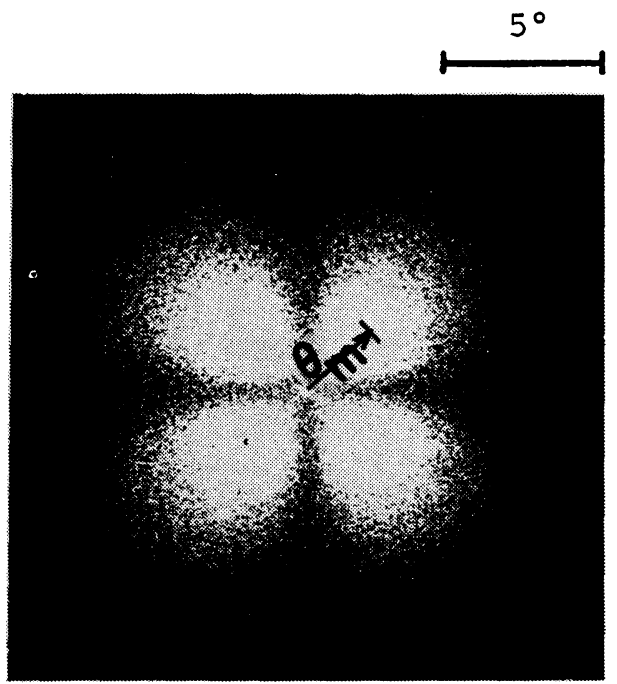

Figure 1. Typical $H_{\mathrm{V}}$ scattering from spherulites. The angle $\theta_{\mathrm{m}}$ is the scattering angle at which the spherulitic scattering intensities become maximum. 
T. Hashimoto, K. Yamaguchi, and H. Kawai

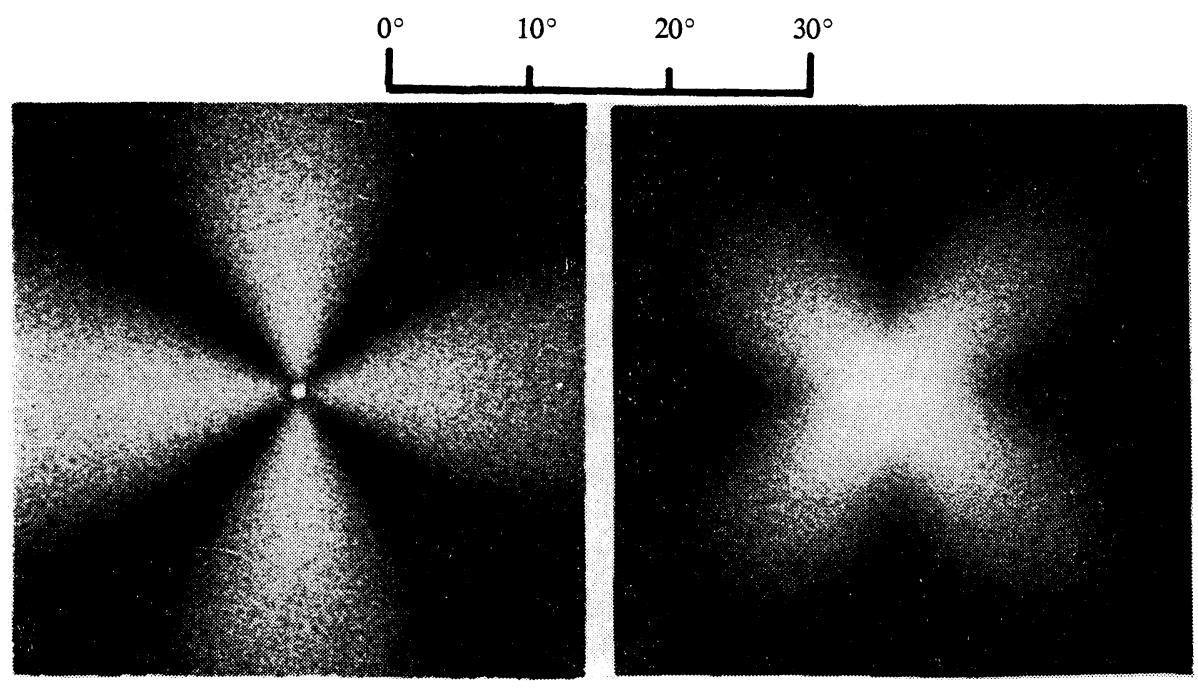

(a)

(b)

Figure 2. Typical $H_{\mathrm{V}}$ scattering from anisotropic rodlike particles. (a) + -type and (b) $\times$-type intensity distributions observed for poly(tetrafluoroethylene).

spherulite assumed to be spherical, and $\lambda$ is the wavelength of light in the medium. The effect of truncation of spherulites on $\theta_{\max }$ was also included recently for a case of random distribution of locations of the nuclei. ${ }^{4}$

In contrast to the spherulitic scattering, the problem of rodlike scattering shown in Figure 2 is relatively unsolved. The rodlike scattering differs from the spherulitic scattering in that the rodlike scattering generally does not exhibit distinct scattering maximum as shown in Figure 1 for the spherulitic scattering.

This is due to the manner of orientation correlation of the optical axes (or chain axes) of anisotropic scattering elements as schematically illustrated in Figures $3(a)$ and $3(b)$. In case of

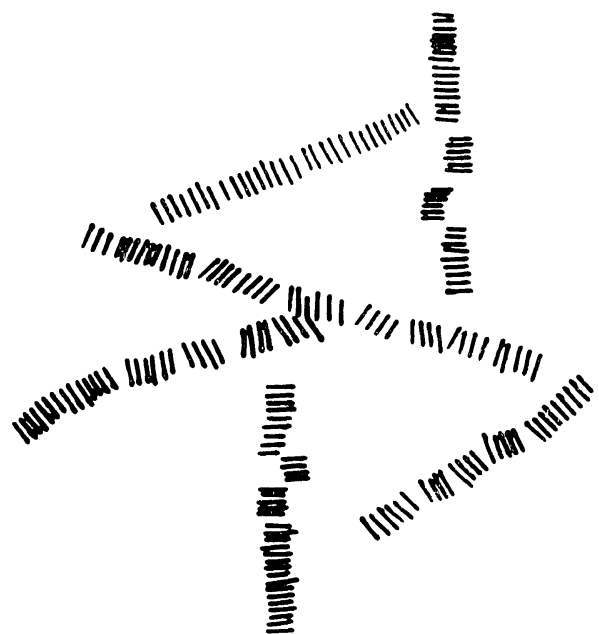

(a)

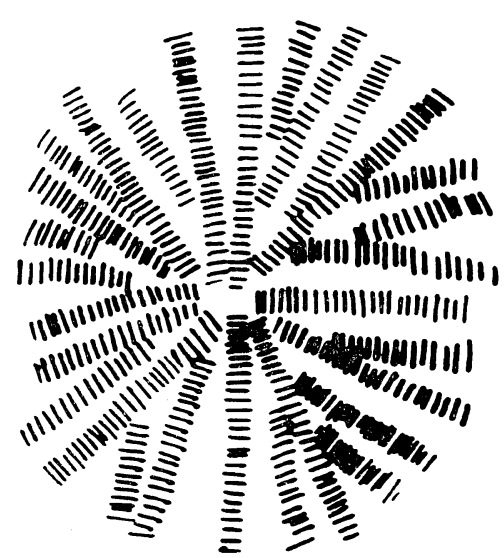

(b)

Figure 3. Schematic representations of orientation fluctuation and correlation of optical axes (indicated by lines) of anisotropic scattering elements; (a) rodlike orientation correlation, and (b) spherulitic orientation correlation. 
the spherulites the amplitudes of scattering from different parts of the spherulite tend to cancell out at very small angles due to symmetry of spherulites in terms of optical axis orientation and distribution of scattering elements. The degree of the cancellation depends upon the internal disorders of spherulite ${ }^{3}$ and deviation of spherulite shape from sphere or disk (twodimensional spherulite). ${ }^{2}$ The cancellation of the scattering amplitudes at the small angles does not occur for the scattering from an isolated particle or a dilute dispersion of the particles having rodlike orientation correlation. Generally, the spatial arrangement of the rodlike scattering particles is disordered with respect to interparticle distances and relative orientation. This results in the observed intensity distribution of rodlike scattering so that the scattered intensity is maximum at the scattering angle $\theta=0^{\circ}$ and continuously decreases with further increase in $\theta$.

There is no convenient relationship by which the size of the rodlike structure can be estimated, as in the case of eq 1 for the spherulitic scattering. Generally, the size of the rodlike particles can be obtained by fitting the experimentally measured scattering curve with that calculated based on a particular model for the scattering entity.

We have recently proposed a more specific way for estimating the size of the rodlike particles for a particular case in which the anisotropic rods having infinitesimally thin lateral dimensions are randomly dispersed in a plane perpendicular to propagation direction of the incident beam. ${ }^{5}$ The principle for measurement is based upon the fact that relative intensity distribution of the arithmetic average of $H_{\nabla}$ scattered intensities at azimuthal angle $\mu=0^{\circ}$ and $45^{\circ}$ is independent of orientation of optical axis of anisotropic scattering elements within the particle but dependent only upon length of the rodlike particle. The average intensity decreases according to the $W^{-1}$ dependence at high scattering angles where $W=2 \pi(L / \lambda) \sin \theta$, and $L$ is the length of the particle. The critical angle $\left(\theta^{*}\right.$ or $\left.W^{*}\right)$ above which the $W^{-1}$ dependence can be applied gives a relationship to estimate the length $L$.

$$
W^{*}=2 \pi(L / \lambda) \sin \theta^{*}=15
$$

In this paper we consider a generalization of our earlier treatment so as to allow estimation of the length and the radius of gyration of lateral cross-section for the optically anisotropic rodlike particles randomly dispersed in two- and threedimensional spaces. This is to generalize well established principles for the scattering from random assembly of isotropic rodlike particles ${ }^{6-8}$ to the scattering from anisotropic rodlike particles.

We shall restrict our treatment to the case in which interparticle interference is negligible. The assumption is good if the rodlike particles exist as a dilute suspension or if the scattering is detected at a particular range of scattering angles, i.e, at sufficiently large angles so that the interference function is close to unity. We shall assume that the size of the rod is monodisperse for simplicity and for a purpose of qualitative measurement of the size and that the effect of multiple scattering can be neglected. The effect of the multiple scattering on the rodlike scattering was recently taken into account by Prud'homme. ${ }^{9}$ Our treatment may be easily generalized to the case where the rod-size is polydisperse.

\section{THEORETICAL BACKGROUND; ESTIMATION OF SIZE PARAMETERS FOR ISOTROPIC RODLIKE PARTICLES}

Let us consider scattering from an isolated rodlike particle oriented at the particular angles $\alpha_{1}$ and $\beta_{1}$ with respect to the reference axis (Figure 4). The particle has uniform polarizability $\alpha_{\mathrm{p}}$, length $L$ and radius $R$ and is dispersed

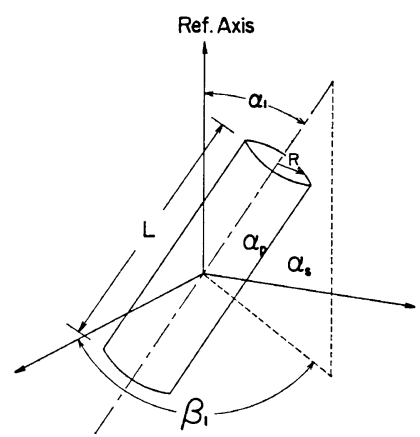

Figure 4. An isotropic rodlike particle having radius $R$, length $L$, and polarizability $\alpha_{\mathrm{p}}$ oriented at a particular angles $\alpha_{1}$ and $\beta_{1}$ with respect to a reference coordinate. 
in an isotropic medium of polarizability $\alpha_{\mathrm{s}}$. If the reference axis is set parallel to the scattering vector $\boldsymbol{h}$, the scattering amplitude $E\left(\alpha_{1}, \beta_{1}\right)$ from the particle is then given by,

$$
\begin{aligned}
& E\left(\alpha_{1}, \beta_{1}\right) \\
& \quad \sim\left(\alpha_{\mathrm{p}}-\alpha_{\mathrm{s}}\right) \int_{v} \exp [i(\boldsymbol{h} \cdot \boldsymbol{r})] \mathrm{d} \boldsymbol{r} \\
& \quad=2 V\left(\alpha_{\mathrm{p}}-\alpha_{\mathrm{s}}\right) \frac{\sin \left(h L / 2 \cos \alpha_{1}\right)}{h L / 2 \cos \alpha_{1}} \frac{J_{1}\left(h R \sin \alpha_{1}\right)}{h R \sin \alpha_{1}}
\end{aligned}
$$

where $V$ is the volume of the particle, $h$ and $h$ are the scattering vector and reduced scattering angle as defined below, respectively, and $J_{1}(x)$ is the first kind Bessel function of the order one.

$$
\begin{aligned}
& \boldsymbol{h}=(2 \pi / \lambda)\left(\boldsymbol{s}-\boldsymbol{s}_{0}\right) \\
& h=(4 \pi / \lambda) \sin (\theta / 2)
\end{aligned}
$$

The vectors $s$ and $s_{0}$ are the unit vectors parallel to the propagation direction of the scattered and the incident beams, respectively.

If the particle is randomly oriented with respect to the angle $\alpha_{1}$ and $\beta_{1}$, the scattered intensity should be averaged over all possible values of $\alpha_{1}$ and $\beta_{1}$ to result in,

$$
\begin{aligned}
I(h) \sim & 4 V^{2}\left(\alpha_{\mathrm{p}}-\alpha_{\mathrm{s}}\right)^{2} \int_{0}^{\pi} \frac{\sin ^{2}\left(h L / 2 \cos \alpha_{1}\right)}{\left(h L / 2 \cos \alpha_{1}\right)^{2}} \\
& \times \frac{J_{1}^{2}\left(h R \sin \alpha_{1}\right)}{\left(h R \sin \alpha_{1}\right)^{2}} \sin \alpha_{1} \mathrm{~d} \alpha_{1}
\end{aligned}
$$

The problems are thus reduced to solving eq 6 numerically and to fit the curve to the experimental intensity distribution so as to estimate the size of the particle. However, a more specific analysis can be applied for a special case in which the dimension of the particle in one direction is extremely large relative to that in the other direction.

If $R \ll L$, then angular dependence of the term $J_{1}^{2}\left(h R \sin \alpha_{1}\right) /\left(h R \sin \alpha_{1}\right)^{2}$ is negligibly small compared with that of the term $\sin ^{2}\left(h L / 2 \cos \alpha_{1}\right) /$ $\left(h L / 2 \cos \alpha_{1}\right)^{2}$ so that the former may be set constant,

$$
\frac{J_{1}{ }^{2}\left(h R \sin \alpha_{1}\right)}{\left(h R \sin \alpha_{1}\right)^{2}} \approx \frac{J_{1}{ }^{2}(h R)}{(h R)^{2}} \approx \exp \left[-(h R)^{2} / 4\right]
$$

From eq 6 and 7 , it follows that

$$
\begin{aligned}
& I(h) \approx 4 V^{2}\left(\alpha_{\mathrm{p}}-\alpha_{\mathrm{s}}\right)^{2} / \exp \left[-(h R)^{2} / 4\right] I_{1}(h) \\
& I_{1}(h)=\int_{0}^{\pi} \frac{\sin ^{2}\left(h L / 2 \cos \alpha_{1}\right)}{\left(h L / 2 \cos \alpha_{1}\right)^{2}} \sin \alpha_{1} \mathrm{~d} \alpha_{1}
\end{aligned}
$$

where $I_{1}(h)$ is associated with scattering from the randomly oriented one-dimensional rod, i.e., the rod having $R=0$.

The integral of eq 9 can be solved analytically to give

$$
I_{1}(h)=\frac{S i U}{(U / 2)}-\frac{\sin ^{2}(U / 2)}{(U / 2)^{2}}
$$

where $S i U$ is the sine function defined by

$$
\begin{gathered}
S i U=\int_{0}^{U}(\sin X) / X \mathrm{~d} X \\
U=4 \pi(L / \lambda) \sin (\theta / 2)
\end{gathered}
$$

The intensity function $I_{1}(h)$ is well analyzed and the two limiting cases are of importance here to evaluate the rod length $L$;

(i) At small $h$ value

The function $I_{1}(h)$ is given by a Gaussian curve as shown in Figure 5,

$$
I_{1}(h) \sim \exp \left(-L^{2} h^{2} / 36\right)
$$

Therefore, the slope of the Guinier plot $\ln I_{1}(h)$ vs. $h^{2}$ yields the estimated value of $L$. However, in order to obtain a meaningful value for $L$, one must be sure that the particles are in a dilute system so that the interparticle interference can be neglected.

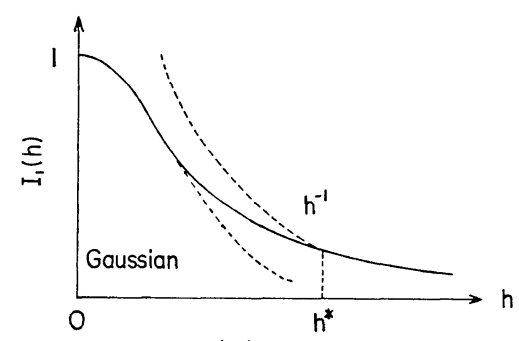

(a)

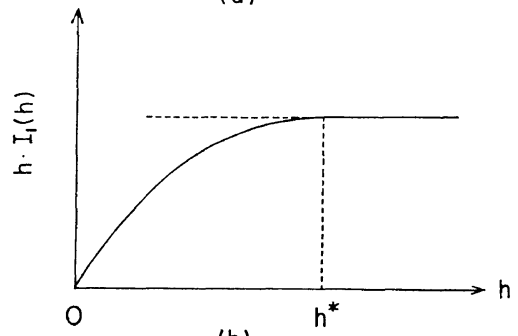

(b)

Figure 5. A schematic representation of the intensity distribution (a) $I_{1}(h)$ and (b) $h I_{1}(h)$ from the randomly oriented one-dimensional rod, and the critical angle $h^{*}$ above which the intensity varies with the $h^{-1}$ dependence. 
Light Scattering from Anisotropic Rodlike Particles

(ii) At a large $h$ value greater than the critical value $h^{*}$

The function $I_{1}(h)$ decreases with increasing $\theta$ according to the $h^{-1}$ dependence, i.e.,

$$
\begin{gathered}
I_{1}(h) \sim(h L)^{-1} \equiv U^{-1} \quad \text { for } \quad U \geq U^{*} \\
U^{*}=4 \pi(L / \lambda) \sin \left(\theta^{*} / 2\right)=L h^{*}
\end{gathered}
$$

The critical reduced scattering angle $U^{*}$ can be theoretically evaluated from the plot of $U I_{1}(U)$ $v s$. $U$. Then the plot of $h I_{1}(h) v s$. $h$ of the type as shown in Figure 5(b) on the measured intensity gives the critical scattering angle $\theta^{*}$ or $h^{*}$.

Thus by using eq 14 , one can evaluate the rod length $L$. The method of evaluation utilizes the intensity distribution at large scattering angles, so that the evaluated size $L$ is less sensitive to the interparticle interference effect than the value obtained by the Guinier plot at small scattering angles. The drawback of this method arises from the fact that one has to measure generally weak intensities at large angles.

The intensity $I(h)$ at even large scattering angles deviates from the $h^{-1}$ dependence, the deviation being dependent upon the front factor $\exp \left[-(h R)^{2} / 4\right]$ of eq 8 . Upon increasing the lateral size $R$ or increasing $h$, the intensity tends to decrease more rapidly than the $h^{-1}$ dependence. From eq 8 and 13, it follows that

$$
\ln h I(h)=\text { (const.) }-\left(R^{2} / 4\right) h^{2}
$$

Thus the Guinier plot of the type $\ln h I(h) v s . h^{2}$ gives a value for $R$ from its slope. The equation can be modified to a more general case in which the shape of the cross section is other than circle, i.e.,

$$
\ln h I(h)=\text { (const. })-\left(R_{\mathrm{q}}{ }^{2} / 2\right) h^{2}
$$

where $R_{\mathrm{q}}$ is the radius of gyration of crosssectional area of the rod-like particle.

Similar relationships can be established for the disklike particles for which $R \gg L$ in eq 6 . In this case eq 12 is modified to

$$
I_{2}(h) \sim \exp \left(-\frac{1}{6} R^{2} h^{2}\right)
$$

Equations 13 and 14 are also modified to

$$
\begin{gathered}
I_{2}(h) \sim(h R)^{-2} \equiv U_{\mathrm{D}}^{-2} \quad \text { for } \quad U_{\mathrm{D}} \geq U_{\mathrm{D}}{ }^{*} \\
U_{\mathrm{D}}{ }^{*}=4 \pi(R / \lambda) \sin \left(\theta^{*} / 2\right)=R h^{*}
\end{gathered}
$$

and finally eq 15 and 16 should be modified to

$$
\begin{aligned}
& \left.\ln h^{2} I(h)=\text { (const. }\right)-\left(L^{2} / 12\right) h^{2} \\
& \left.\ln h^{2} I(h)=\text { (const. }\right)-\left(R_{\mathrm{q}}{ }^{2} / 2\right) h^{2}
\end{aligned}
$$

In the next section, we will generalize the principles for evaluating the size of anisotropic rodlike particle randomly distributed in two- and three-dimensional sample spaces.

\section{EXTENSION TO ANISOTROPIC RODLIKE PARTICLES}

Figure 6 shows the optically anisotropic rodlike particle whose axis is designated by the unit vector $\boldsymbol{r}_{1}$ oriented at the particular angles $\alpha$ and $\beta$ with respect to the Cartesian coordinate fixed to the apparatus. The unit vectors $s_{0}$ and $s^{\prime}$ are parallel to the propagation direction of the incident and scattered beams; $\theta$ and $\mu$ are, the scattering and azimuthal angles respectively of the observed scattered intensity; the vectors $\boldsymbol{i}, \boldsymbol{j}$, and $\boldsymbol{k}$ are the unit vectors parallel to $x, y, z$-axes, respectively. The scattering elements are assumed to have optically uniaxial anisotropy with polarizabilities $\alpha_{\|}$and $\alpha_{\perp}$, respectively, parallel and perpendicular to the optical axis designated by the unit vector $d$.

Previously, two types of rod models were considered in terms of the manner of orientation of the principal optical axis $\boldsymbol{d}$ within the rod; ${ }^{5,10}$ (i) the rod of model $\mathrm{A}$ for which the optical axis is assumed to form a polar angle $\omega_{0}$ with the vector $\boldsymbol{r}_{1}$ and to lie in a particular plane specified by an azimuthal angle $\gamma$, and (ii) the rod of model B for which the optical axis is

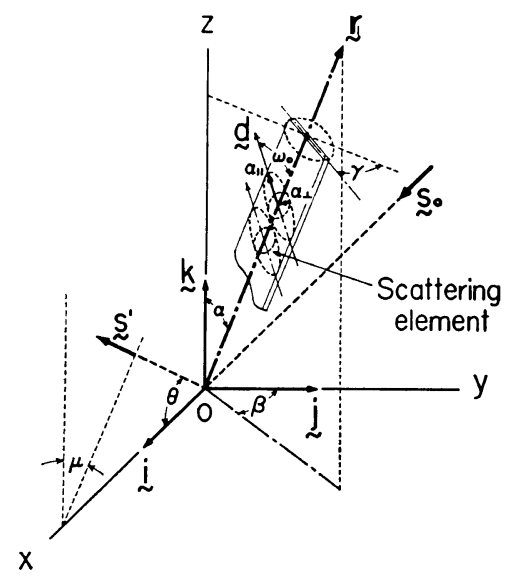

Figure 6. An anisotropic rodlike particle. 
assumed to orient in cylindrical symmetry with a constant value of $\omega_{0}$ but randomly varying $\gamma$. For the former model, the scattering were calculated for two special cases, i.e., (i) $\gamma$ being random for three-dimensional random orientation $r_{1}$ and (ii) $\gamma=0$ for two-dimensional random orientation $r_{1}$ with $\beta=0$.

A difficulty in estimating the size of the anisotropic rodlike particle compared with that of the isotropic rodlike particle generally arises from the fact that the angular distribution of scattering from the anisotropic rod depends upon the manner of orientation of the optical axes within the rod as well as the size of the rod as discussed in the previous section.

Consider the scattering under $H_{\mathrm{V}}$ polarization in which scattering from vertically polarized incident beam is detected through horizontally polarized analyzer placed in front of the detecting device. For the rod of length $L$ and radius $R$, the scattering amplitude from a particle at a given orientation is given by

$$
\begin{aligned}
E_{\mathrm{H}_{\mathrm{V}}}\left(\alpha_{1}\right) & =K(\boldsymbol{M} \cdot \boldsymbol{O}) \int_{V} \exp [i(\boldsymbol{h} \cdot \boldsymbol{r})] \mathrm{d} \boldsymbol{r} \\
& =2 V K(\boldsymbol{M} \cdot \boldsymbol{O}) \frac{\sin \left(h L / 2 \cos \alpha_{1}\right)}{\left(h L / 2 \cos \alpha_{1}\right)} \frac{J_{1}\left(h R \sin \alpha_{1}\right)}{\left(h R \sin \alpha_{1}\right)}
\end{aligned}
$$

where $\alpha_{1}$ is the angle between the scattering vector $\boldsymbol{h}$ and the rod axis $\boldsymbol{r}_{1}$, and $(\boldsymbol{M} \cdot \boldsymbol{O})$ is the induced dipole moment under the given polarization condition. The induced dipole moment reduces to zero for the $H_{\mathrm{V}}$ scattering and to $\left(\alpha_{\mathrm{p}}-\alpha_{\mathrm{s}}\right)$, as in eq 3 for the scattering from isotropic rods under parallel polarizers in which polarization direction of the incident field is parallel to that of the analyzer.

On averaging the scattered intensity over all possible orientation of the rod, one obtains the $H_{\mathrm{V}}$ scattered intensity from the anisotropic rods. The scattered intensity generally depends on $\mu$ as well as $h$ (or $\theta$ ) due to the anisotropy. If $L \gg R$, it follows that

$$
\begin{aligned}
I_{\mathrm{H}_{\mathrm{V}}}(h, \mu) & =\frac{1}{2} \int_{0}^{\pi}\left|E_{\mathrm{H}_{\mathrm{V}}}\left(\alpha_{1}\right)\right|^{2} \sin \alpha_{1} \mathrm{~d} \alpha_{1} \\
& \approx \text { (const. }) \exp \left(-h^{2} R^{2} / 4\right) \mathscr{F}_{\mathrm{H}_{\mathrm{V}}}(h, \mu)
\end{aligned}
$$

as in the scattering from isotropic rods. $\mathscr{F}_{\mathrm{H}_{\mathrm{V}}}$ is the scattering from one-dimensional and an- isotropic rods. The intensity formulae for the rods of models A and B were previously given; For the rod of model $A^{11}$

$$
\begin{aligned}
& \mathscr{J}_{\mathrm{H}_{\mathrm{V}}}(h, \mu) \\
& =\int_{\alpha=0}^{\pi} \int_{\beta=0}^{2 \pi}\left\langle(\boldsymbol{M} \cdot \boldsymbol{O})^{2}\right\rangle_{\gamma} \frac{\sin ^{2}(h L / 2 \cos \alpha)}{(h L / 2 \cos \alpha)^{2}} \sin \alpha \mathrm{d} \alpha \mathrm{d} \beta \\
& =(\operatorname{const} .)\left\{\frac { 3 } { 8 } \left[35 \cos ^{4}(\theta / 2) \sin ^{2} 2 \mu+4\right.\right. \\
& \left.\quad-20 \cos ^{2}(\theta / 2)\right] P_{4}\left(\cos \omega_{0}\right)(35 A-30 B+3 C) \\
& \left.\quad+10\left[3 \cos ^{2}(\theta / 2)-2\right] P_{2}\left(\cos \omega_{0}\right)(3 B-C)+28 C\right\}
\end{aligned}
$$

For the rod of model $B^{10}$

$$
\begin{aligned}
& \mathscr{F}_{\mathrm{H}_{\mathrm{v}}}(h, \mu) \\
& =\int_{\alpha=0}^{\pi} \int_{\beta=0}^{2 \pi}\left\langle(M \cdot O)^{2}\right\rangle_{r} \frac{\sin ^{2}(h L / 2 \cos \alpha)}{(h L / 2 \cos \alpha)^{2}} \sin \alpha \mathrm{d} \alpha \mathrm{d} \beta \\
& =(\text { const. })\left[P_{2}\left(\cos \omega_{0}\right)\right]^{2}\left\{(35 A-30 B+3 C) \cos ^{4}\right. \\
& \quad \times(\theta / 2) \sin ^{2} 2 \mu-4(5 A-6 B+C) \cos ^{2}(\theta / 2) \\
& \quad+4(A-2 B+C)\}
\end{aligned}
$$

where $A, B$, and $C$ are the function of rod length $L$, and $P_{2}$ and $P_{4}$ are the second and forth order Legendre functions, respectively,

$$
\begin{aligned}
& A=\frac{1}{3 U_{1}^{2}}-\frac{2 U_{1}^{2}-1}{4 U_{1}^{5}} \sin 2 U_{1}-\frac{1}{2 U_{1}^{4}} \cos 2 U_{1} \\
& B=\frac{1}{U_{1}^{2}}-\frac{\sin 2 U_{1}}{2 U_{1}^{3}} \\
& C=2\left[\frac{S i\left(2 U_{1}\right)}{U_{1}}-\frac{\sin ^{2} U_{1}}{U_{1}^{2}}\right] \\
& U=2 U_{1}=4 \pi(L / \lambda) \sin (\theta / 2)
\end{aligned}
$$

For either type of rod model, a relative intensity distribution of $\mathscr{I}_{\mathrm{H}_{\mathrm{V}}}\left(h, \mu=0^{\circ}\right)-\mathscr{I}_{\mathrm{H}_{\mathrm{V}}}(h$, $\left.\mu=45^{\circ}\right) \mid$ with $\theta$ or $h$ is independent of the manner of the orintation of optical axis but dependent only upon length of the rod. If the intensity difference is defined by

$$
D_{\mathrm{H}_{\mathrm{V}}}(\theta) \equiv\left|\mathscr{J}_{\mathrm{H}_{\mathrm{V}}}\left(\mu=0^{\circ}\right)-\mathscr{I}_{\mathrm{H}_{\mathrm{V}}}\left(\mu=45^{\circ}\right)\right|
$$

then,

$$
\begin{aligned}
D_{\mathrm{H}_{\mathrm{V}}}(\theta)= & (\text { const. }) P_{4}\left(\cos \omega_{0}\right) \cos ^{4}(\theta / 2) \\
& \times(35 A-30 B+3 C)
\end{aligned}
$$

for the model $\mathrm{A}$ rod and for the model $\mathrm{B}$ rod, ${ }^{12}$

$$
\begin{aligned}
D_{\mathrm{H}_{\mathrm{v}}}(\theta)= & (\text { const. })\left[P_{2}\left(\cos \omega_{0}\right)\right]^{2} \cos ^{4}(\theta / 2) \\
& \times(35 A-30 B+3 C)
\end{aligned}
$$

At large scattering angles, intensity distribu- 
tion of the intensity difference $D_{\mathrm{H}_{\mathrm{V}}} / \cos ^{4}(\theta / 2)$ approaches that of $C$ in eq 24 . Therefore, the intensity distribution $D_{\mathrm{H}_{\mathrm{V}}}$ at a large $\theta$ becomes identical to that for the isotropic rod, i.e., $I_{1}(h)$ given by eq 10 . Consequently one can follow the same procedure as that developed for estimating the size of the isotropic rod with the intensity difference $D_{\mathrm{H}_{\mathrm{v}}}(\theta) / \cos ^{4}(\theta / 2)$.

$$
D_{\mathrm{H}_{\mathrm{V}}}(U) / \cos ^{4}(\theta / 2)=\text { (const.) } U^{-1} \quad \text { for } \quad U \geq U^{*}
$$

where $U^{*}$ is given by eq 14 , from which the length of the anisotropic rodlike particle can be estimated as in the case of the isotropic rod.

From eq 21 it follows that

$$
\begin{aligned}
\left(I_{\mathrm{H}_{\mathrm{V}}}\right)_{\mathrm{dif}} & \equiv\left|I_{\mathrm{H}_{\mathrm{V}}}\left(h, \mu=45^{\circ}\right)-I_{\mathrm{H}_{\mathrm{V}}}\left(h, \mu=0^{\circ}\right)\right| \\
& =\text { (const. }) \exp \left(-h^{2} R^{2} / 4\right) D_{\mathrm{H}_{\mathrm{V}}}(h)
\end{aligned}
$$

At a relatively small $h$ value, the factor $\exp \left(-h^{2} R^{2} / 4\right)$ is close to unity, so that $\left(I_{\mathrm{H}_{\mathrm{v}}}\right)_{\mathrm{dif}}$ decreases with increasing $h$ with the $U^{-1}$ or $h^{-1}$ dependence. However, at a larger $h$ value the factor associated with lateral dimensions affects the intensity distribution so that the intensity decreases with increasing $h$ much faster than the $U^{-1}$ or $h^{-1}$ dependence. Therefore, the angular interval where the $h^{-1}$ dependence is valid depends strongly upon a relative size of $L$ and $R$.

From eq 28 and 29, it follows that

$$
\begin{aligned}
\ln h\left(I_{\mathrm{H}_{\mathrm{v}}}\right)_{\mathrm{dif}} / \cos ^{4}(\theta / 2) & =\text { (const. })-\left(R^{2} / 4\right) h^{2} \\
& =\text { (const. })-\left(R_{\mathrm{q}}{ }^{2} / 2\right) h^{2}
\end{aligned}
$$

Therefore, one can evaluate the radius of the gyration of a cross sectional area of the anisotropic rodlike particle exactly in the same way as in the isotropic rod with $\left(I_{\mathrm{H}_{\mathrm{v}}}\right)_{\mathrm{dif}}{ }^{13}$

A similar procedure may be applied to the anisotropic disklike particle.

So far we have assumed that the anisotropic rodlike particles are oriented randomly in a three-dimensional space. Occasionally, the experimental systems may have planar orientation of the particles. The treatment described above can be easily extended to a system in which the particles are randomly oriented in a $Y O Z$ plane normal to propagation direction of the incident beam. It can be shown that for such a system, eq 21 is still valid. The intensity functions $\mathscr{I}_{\mathrm{H}_{\mathrm{V}}}$ for the one-dimensional rod oriented randomly in the two-dimensional space were previously derived for various cases. ${ }^{5}$

The intensity difference $D_{\mathrm{H}_{\mathrm{V}}}(\theta)$ for the twodimensional orientation of the rod is given by

$$
D_{\mathrm{H}_{\mathrm{v}}}(W)=\text { (const.) } \cos 4 \omega_{0} F(W) / W^{2}
$$

for the rod of model $\mathrm{A}$, and

$$
\left.D_{\mathrm{H}_{\mathrm{V}}}(W)=\text { (const. }\right)\left[P_{2}\left(\cos \omega_{0}\right)\right]^{2} F(W) / W^{2}
$$

for the rod of model B. The function $F(W)$ depends only upon the length of the rod,

$$
\begin{aligned}
F(W)= & -12 J_{2}(W)+W J_{1}(W)-4 J_{0}(W) \\
& +4-W I_{2}(W)
\end{aligned}
$$

where

$$
I_{2}(W)=\int_{0}^{W} J_{4}(x) \mathrm{d} x, \quad W=2 \pi(L / \lambda) \sin \theta
$$

$J_{i}(W)$ is a Bessel function of the first kind of order $i$. The equations are derived for the homogeneous rod. It can be shown that, at large scattering angles greater than $W^{*}$,

$$
\begin{gathered}
D_{\mathrm{H}_{\mathrm{v}}}(W)=\text { (const.) } W^{-1} \text { for } W \geq W_{\mathrm{d}}{ }^{*} \\
W_{\mathrm{d}}{ }^{*}=2 \pi(L / \lambda) \sin \theta^{*}
\end{gathered}
$$

The critical value of $W\left(=W_{\mathrm{d}}{ }^{*}\right)$ can be evaluated numerically, and measurement of the critical scattering angle $\theta^{*}$ above which the intensity $\left(I_{\mathrm{H}_{\nabla}}\right)_{\text {dif }}$ varies with the $h^{-1}$ dependence gives an estimated value for $L$. It is obvious that eq 29 and 30 are still valid if eq 30 is slightly modified,

$$
\left.\ln h\left(I_{\mathrm{H}_{\mathrm{v}}}\right)_{\mathrm{dif}}=\text { (const. }\right)-\left({R_{\mathrm{q}}}^{2} / 2\right) h^{2}
$$

Thus one can estimate $R_{\mathrm{q}}$ exactly in the same way as described before.

For the special case where the particles are randomly oriented in the two-dimensional plane one can also make use of an averaged intensity $\left(I_{\mathrm{H}_{\mathrm{V}}}\right)_{\mathrm{aV}}$ as an intensity which depends only upon the size of the rod. As already given in the previous paper, ${ }^{5}$

$$
\begin{aligned}
\left(I_{\mathrm{H}_{\mathrm{v}}}\right)_{\mathrm{av}} & =\left[I_{\mathrm{H} \mathrm{v}}\left(\mu=0^{\circ}\right)+I_{\mathrm{H}_{\mathrm{v}}}\left(\mu=45^{\circ}\right)\right] / 2 \\
& =\text { (const. })\left[I_{1}(W)-J_{1}(W)\right] / W
\end{aligned}
$$

\section{RESULTS OF NUMERICAL CALCULATIONS}

Figure 7 shows the calculated results for a random assembly of anisotropic one-dimensional rods in three-dimensional space. Both $U \cdot\left(I_{\mathrm{H}_{\mathrm{v}}}\right)_{\mathrm{dif}} /$ 


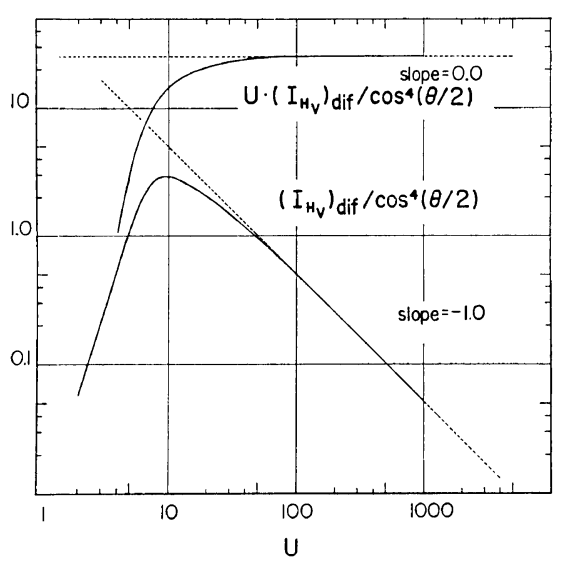

Figure 7. Plots of $U \cdot\left(I_{\mathrm{H}_{\mathrm{V}}}\right)_{\mathrm{dif}} / \cos ^{4}(\theta / 2)$ and $\left(I_{\mathrm{H}_{\mathrm{V}}}\right)_{\mathrm{dif}} /$ $\cos ^{4}(\theta / 2)$ vs. $U$ for the anisotropic one-dimensional rod in three-dimensional space. $\left(I_{\mathrm{H}_{\mathrm{V}}}\right)_{\mathrm{dif}}=\mid I_{\mathrm{H}_{\mathrm{V}}}(\mu=$ $\left.0^{\circ}\right)-I_{\mathrm{H}_{\mathrm{V}}}\left(\mu=45^{\circ}\right) \mid, U=4 \pi(L / \lambda) \sin (\theta / 2)$ and $U^{*}=50$.

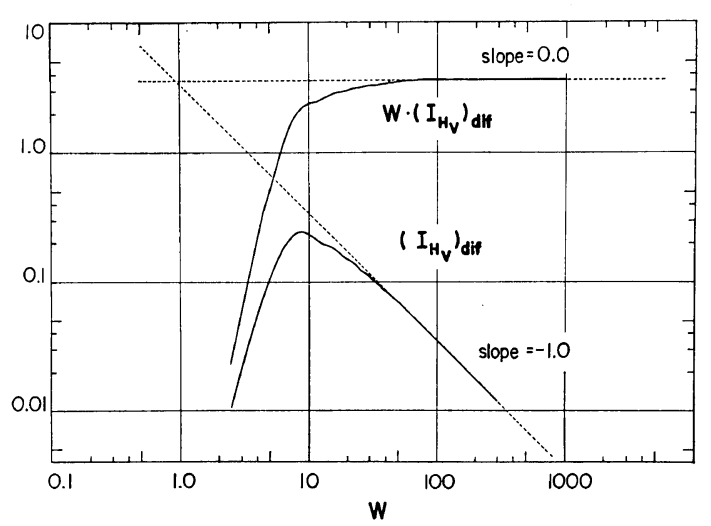

Figure 8. Plots of $W \cdot\left(I_{\mathrm{H}_{\nabla}}\right)_{\mathrm{dif}}$ and $\left(I_{\mathrm{H}_{\mathrm{V}}}\right)_{\mathrm{d} \text { if }} v s$. $W$ for the anisotropic one-dimensional rod in the twodimensional space. $W=2 \pi(L / \lambda) \sin \theta$ and $W^{*}=50$.

$\cos ^{4}(\theta / 2)$ and $\left(I_{\mathrm{H}_{\nabla}}\right)_{\mathrm{dif}} / \cos ^{4}(\theta / 2)$ vs. $U$ demonstrate that the intensity difference of $H_{\mathrm{V}}$ scattering at $\mu=0^{\circ}$ and $45^{\circ}$ corrected by the factor $\cos ^{4}(\theta / 2)$ decreases with an increase in the scattering angle according to the $U^{-1}$ dependence at scattering angles satisfying the condition $U \geq U^{*}$

$$
U^{*}=4 \pi(L / \lambda) \sin \left(\theta^{*} / 2\right)=50
$$

Similarly, the intensity difference $\left(I_{\mathrm{H}_{\mathrm{V}}}\right)_{\mathrm{dif}}$ for a random assembly of anisotropic one-dimensional rods in the two-dimensional plane is shown to vary according to the $W^{-1}$ dependence at $W \geq W_{\mathrm{d}}^{*}$ (Figure 8 ).

$$
W_{\mathrm{d}}^{*}=2 \pi(L / \lambda) \sin \theta^{*}=50
$$

If one measures the average intensity $\left(I_{\mathrm{H}_{\mathrm{V}}}\right)_{\mathrm{av}}$ rather than $\left(I_{\mathrm{H}_{\mathrm{v}}}\right)_{\mathrm{dif}}$ for the assembly of the rods in the two-dimensional plane, the average intensity is also shown to vary with the $W^{-1}$ dependence at $W \geq W_{\mathrm{a}}{ }^{*}$ (Figure 9) as given in a previous paper,

$$
W_{\mathrm{a}}^{*}=2 \pi(L / \lambda) \sin \theta^{*}=15
$$

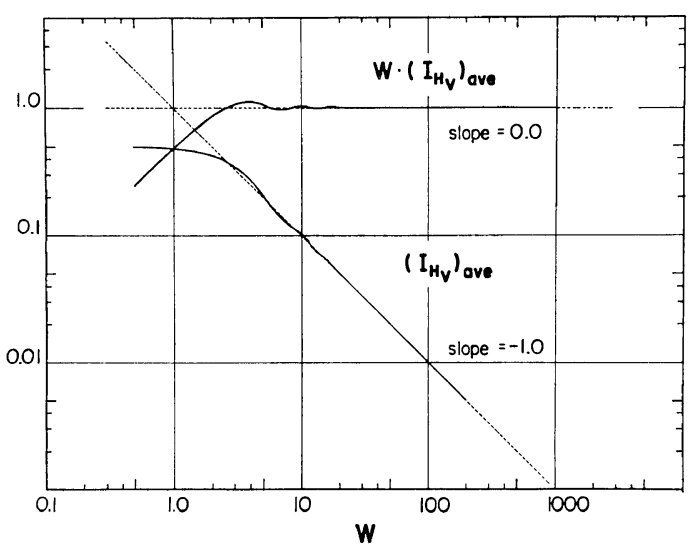

Figure 9. Plots of $W\left(I_{\mathrm{H}_{\mathrm{V}}}\right)_{\mathrm{av}}$ and $\left(I_{\mathrm{H}_{\mathrm{V}}}\right)_{\mathrm{av}} v \boldsymbol{v}$. $W$ for the anisotropic one-dimensional rod in the twodimensional space. $\left(I_{\mathrm{H}_{\mathrm{V}}}\right)_{\mathrm{av}}=\mid I_{\mathrm{H}_{\mathrm{V}}}\left(\mu=0^{\circ}\right)+I_{\mathrm{H}_{\mathrm{V}}}(\mu=$ $\left.45^{\circ}\right) \mid / 2$, and $W^{*}=15$.

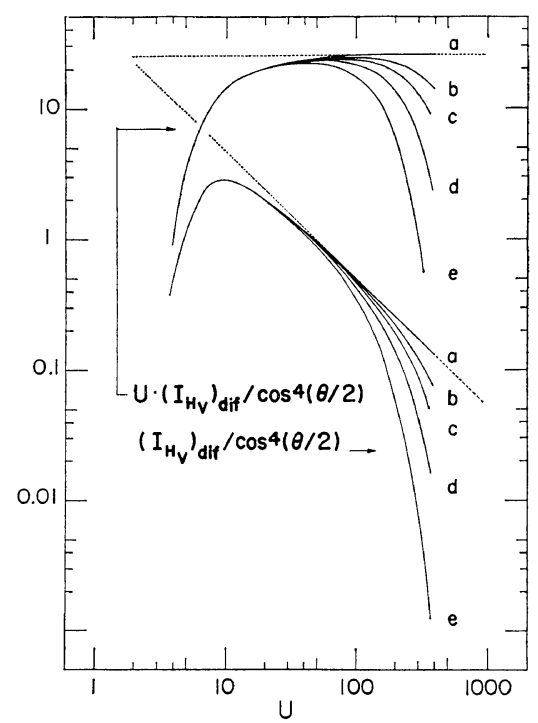

Figure 10. Plots of $U\left(I_{\mathrm{H}_{\mathrm{V}}}\right)_{\mathrm{dif}} / \cos ^{4}(\theta / 2)$ and $\left(I_{\mathrm{H}_{\mathrm{V}}}\right)_{\mathrm{dif}} /$ $\cos ^{4}(\theta / 2)$ for the anisotropic rod in three-dimensional space. The radius of the $\operatorname{rod} R$ is set to be (a) $R / L=0$, (b) $0.68 \times 10^{-2}$, (c) $0.92 \times 10^{-2}$, (d) $1.1 \times 10^{-2}$, and (e) $1.7 \times 10^{-2}$. 
Although the determination of the critical values for $U^{*}, W_{\mathrm{d}}{ }^{*}$ and $W_{\mathrm{a}}{ }^{*}$ involves some uncertainty, these are estimated as values beyond which deviations of the intensities $\left(I_{\mathrm{H}_{\mathrm{V}}}\right)_{\mathrm{dif}} / \cos ^{4}(\theta / 2)$, $\left(I_{\mathrm{H}_{\mathrm{V}}}\right)_{\mathrm{dif}}$ and $\left(I_{\mathrm{H}_{\mathrm{V}}}\right)_{\mathrm{av}}$ from the $U^{-1}$ and $W^{-1}$ dependences become less than $5 \%$. Experimental evaluation of $\theta^{*}$ then yields an estimated length of the rodlike particle.

Figure 10 shows the effect of radius $R$ (radius of gyration $R_{\mathrm{q}}$ of cross-sectional area) on the angular dependence of intensity difference $\left(I_{\mathrm{H}_{\mathrm{v}}}\right)_{\mathrm{dif}} / \cos ^{4}(\theta / 2)$. It is obvious that the intensity first drops in proportion to $U^{-1}$ with an increase in the scattering angle and then more rapidly due to the effect of the finite lateral size. The deviation from the $U^{-1}$ dependence is greater and occurs at smaller scattering angles for the rod with larger lateral size.

\section{DISCUSSIONS; A COMPARISON WITH EXPERIMENTAL RESULTS}

The theoretical results were compared with

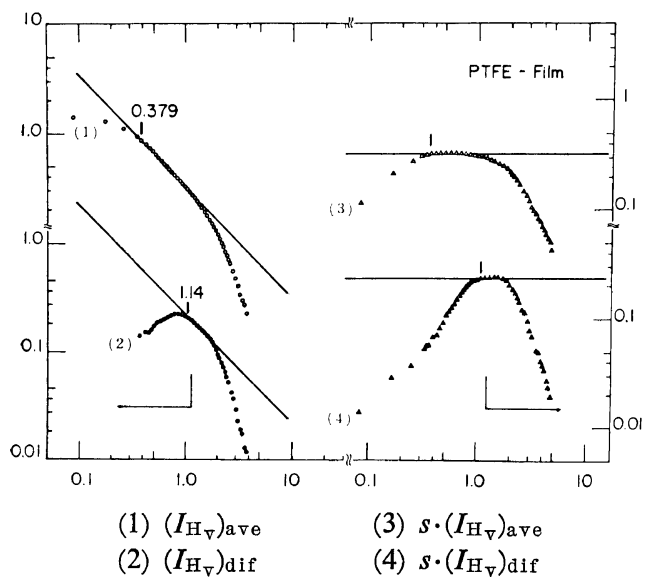

Figure 11. Measured intensity distributions $\left(I_{\mathrm{H}_{\mathrm{V}}}\right)_{\text {av }}$ (curve 1), $\left(I_{\mathrm{H}_{\mathrm{V}}}\right)_{\text {dif }}$ (curve 2), $s\left(I_{\mathrm{H}_{\mathrm{V}}}\right)_{\mathrm{av}}$ (curve 3 ), and $s\left(I_{\mathrm{H}_{\nabla}}\right)_{\text {dif }}$ (curve 4) with $s(=(2 \pi / \lambda) \sin \theta)$ for poly(tetrafluoroethylene) film PTFE-L-ii. scattering from a thin film of poly(tetrafluoroethylene) PTFE-L-ii whose morphology of crystalline superstructure and scattered intensity distributions were described in a previous paper. ${ }^{5}$

Figure 11 shows the plots of $\left(I_{\mathrm{H}_{\mathrm{v}}}\right)_{\mathrm{av}},\left(I_{\mathrm{H}_{\mathrm{V}}}\right)_{\mathrm{dIf}}$, $s\left(I_{\mathrm{H}_{\mathrm{v}}}\right)_{\mathrm{av}}$, and $s\left(I_{\mathrm{H}_{\mathrm{v}}}\right)_{\text {dif }} v s$. $s$ to estimate the length of the rodlike particle $L$. In our previous paper the rodlike particles showed a tendency of the planar orientation so that the intensity distribution $\left(I_{\mathrm{H}_{\mathrm{V}}}\right)_{\mathrm{av}}$ was also considered. From this figure it is apparent that the intensities $\left(I_{\mathrm{H}_{\mathrm{V}}}\right)_{\mathrm{aV}}$ and $\left(I_{\mathrm{H}_{\mathrm{V}}}\right)_{\mathrm{dif}}$ drop with $s^{-1}$ at certain angular intervals. With a further increase in $s$, the intensities drop more rapidly than $s^{-1}$ due to the effect of the lateral size. The critical values of $s^{*}$ were 0.379 and 1.14 for $\left(I_{\mathrm{H}_{\mathrm{V}}}\right)_{\mathrm{av}}$ and $\left(I_{\mathrm{H}_{\mathrm{V}}}\right)_{\mathrm{dif}}$, respectively, from which the values for $L$ were estimated to be 40 and 44 microns, respectively. The small difference in $L$ is due to an error in the evaluated $s^{*}$. It should be noted that the plots of $\left(I_{\mathrm{H}_{\mathrm{v}}}\right)_{\mathrm{dif}} / \cos ^{4}(\theta / 2) v s$. $s$ and $s\left[\left(I_{\mathrm{H}_{\mathrm{v}}}\right)_{\mathrm{dif}} / \cos ^{4}(\theta / 2)\right]$ vs. $s$ yielded the value of $L=44$ microns.

Figure 12 shows the Guinier plot to obtain the lateral size of the rodlike particle. The plot of the type $\ln \left[s\left(I_{\mathrm{H}_{v}}\right)_{a v}\right]$ vs. $h^{2}$ gave $R=1.3$

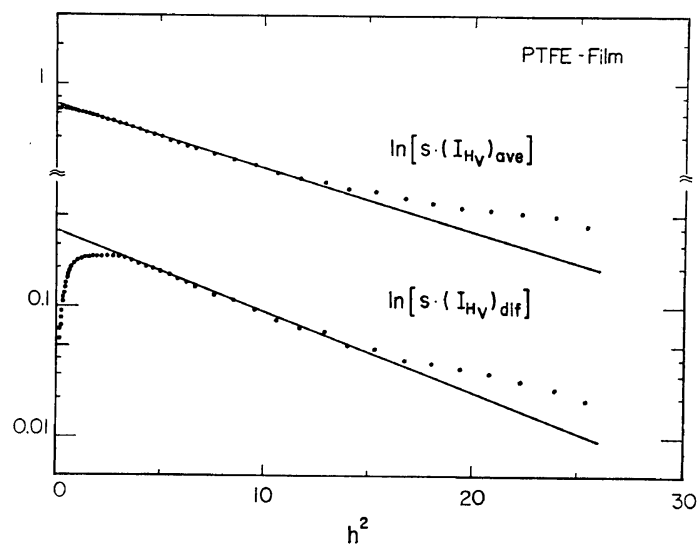

Figure 12. Guinier plots for the cross-sectional area of the rodlike particle for the PTFE-L-ii specimens; $\ln \left[s\left(I_{\mathrm{H}_{\mathrm{V}}}\right)_{\mathrm{av}}\right], \ln \left[s\left(I_{\mathrm{H}_{\mathrm{v}}}\right)_{\mathrm{dif}}\right] v s . h^{2}$.

Table I. Estimated size of anisotropic rodlike particle for the PTFE-L-ii specimens.

\begin{tabular}{lcccc}
\hline & & \multicolumn{2}{c}{ Lateral dimensions } & D \\
\cline { 3 - 5 } & Length, $L$ & $R_{\mathrm{q}}$ & $D$ & \\
\hline $\begin{array}{l}\text { Light scattering measurements } \\
\text { Electron microscopic observations }\end{array}$ & $40-44 \mu$ & $0.9-1.1 \mu$ & $2.6-3.0 \mu$ & $0.06-0.07$ \\
& $40 \mu$ & - & $2 \mu$ & 0.05 \\
\hline
\end{tabular}


microns, and that of $\ln \left[s\left(I_{\mathrm{H}_{\mathrm{v}}}\right)_{\mathrm{dif}}\right]$ vs. $h^{2}$ or $\ln \left[s\left(I_{\mathrm{H}_{\mathrm{v}}}\right)_{\mathrm{dif}} / \cos ^{4}(\theta / 2)\right] v s . h^{2}$ gave $R=1.5$ microns.

Table I shows a comparison of the estimated size of the anisotropic rodlike particle of the PTFE-L-ii specimens with the size observed by electron microscopy. ${ }^{5}$ The estimated length $L$ and diameter $D$ are shown to agree fairly well with the observed values from the micrograph. This good agreement may demonstrate, at least qualitatively, the applicability of the proposed treatment to estimate the size of the anisotropic rodlike particles.

Acknowledgments. A part of this works was supported by a grant from the Takeda Science Foundation and a grant from the Kawakami Memorial Foundation.

\section{REFERENCES AND NOTES}

1. R. S. Stein and M. B. Rhodes, J. Appl. Phys., 31, 1873 (1960).

2. R. E. Prud'homme and R. S. Stein, J. Polym. Sci., Polym. Phys. Ed., 11, 1357, 1633 (1973).

3. D. Y. Yoon and R. S. Stein, ibid., 12, 735, 763
(1974).

4. R.S. Stein, private communication.

5. T. Hashimoto, Y. Murakami, and H. Kawai, J. Polym. Sci., Polym. Phys. Ed., 13, 1613 (1975).

6. O. Kratky and O. Porod, Acta Phys. Austriaca, 2, 133 (1948).

7. G. Porod, ibid., 2, 255 (1948).

8. O. Kratky and G. Porod, J. Polym. Sci., 16, 163 (1955).

9. D. Le'vesque and R. E. Prud'homme, to be published.

10. Y. Murakami, N. Hayashi, T. Hashimoto, and H. Kawai, Polym. J., 4, 452 (1973).

11. M. Moritani, N. Hayashi, A. Utsuo, and $\mathbf{H}$. Kawai, ibid., 2, 74 (1971).

12. If it is known that particles under consideration are the rodlike particles of model $\mathrm{B}$, the relative intensity $\mathscr{I}_{\mathrm{H}_{\mathrm{V}}}(h, \mu)$ itself depends only upon size of the particle $L$. Therefore, it is not necessary to take $D_{\mathrm{H}_{\mathrm{v}}}(\theta)$. It should be noted, however, that the critical angle $U^{*}$ is dependent upon $\mu$.

13. For a qualitative evaluation of $R$ or $R_{\mathrm{q}}$ only, it may be suffice to use a Guinier plot of the type $\ln h I_{\mathrm{H}_{\mathrm{v}}}\left(\mu_{1}\right) v s . h^{2}$ based on $H_{\mathrm{V}}$ scattered intensity at any particular azimuthal angle. 